\title{
Recovery of the mitochondrial COI barcode region in diverse Hexapoda through tRNA-based primers
}

\author{
Doo-Sang Park1, Soo-Jung Suh ${ }^{3}$, Hyun-Woo Oh² and Paul DN Hebert*4
}

\begin{abstract}
Background: DNA barcoding uses a 650 bp segment of the mitochondrial cytochrome c oxidase I (COI) gene as the basis for an identification system for members of the animal kingdom and some other groups of eukaryotes. PCR amplification of the barcode region is a key step in the analytical chain, but it sometimes fails because of a lack of homology between the standard primer sets and target DNA.
\end{abstract}

Results: Two forward PCR primers were developed following analysis of all known arthropod mitochondrial genome arrangements and sequence alignment of the tRNA-W gene which was usually located within 200 bp upstream of the COl gene. These two primers were combined with a standard reverse primer (LepR1) to produce a cocktail which generated a barcode amplicon from 125 of 141 species that included representatives of 121 different families of Hexapoda. High quality sequences were recovered from $79 \%$ of the species including groups, such as scale insects, that invariably fail to amplify with standard primers.

Conclusions: A cocktail of two tRNA-W forward primers coupled with a standard reverse primer amplifies COI for most hexapods, allowing characterization of the standard barcode primer binding region in COI 5 ' as well as the barcode segment. The current results show that primers designed to bind to highly conserved gene regions upstream of COI will aid the amplification of this gene region in species where standard primers fail and provide valuable information to design a primer for problem groups.

\section{Background}

Since 2003, substantial effort has been directed toward the development of a DNA-based identification system for animal life, based upon the analysis of sequence diversity in the $5^{\prime}$ region of the mitochondrial gene, cytochrome $c$ oxidase $1[1,2]$. Termed DNA barcoding, this approach relies upon the PCR amplification of the target gene region and its subsequent sequence characterization. Performance tests on numerous animal groups have established that the system ordinarily works well sequence diversity in the 5 region of COI enables discrimination of more than $98 \%$ of animal species [3,4]. However, past work has also revealed that standard primer cocktails fail to generate a PCR amplicon in certain taxonomic groups. In some cases, amplification success has been so low that researchers have suggested the need to study slower evolving gene regions that can be

* Correspondence: phebert@uoguelph.ca

4 Biodiversity Institute of Ontario, University of Guelph, Guelph, Ontario, N1G 2W1, Canada

Full list of author information is available at the end of the article recovered more easily [5,6]. Because such movement away from COI represents a serious compromise from the standardization that is fundamental to DNA barcoding, there is much incentive to develop new primer sets that enable recovery of the standard barcode region for 'problem' taxonomic groups.

The Arthropoda represent, by far, the most diverse of animal phyla. Although current primer sets generally perform well, there are some groups where barcode recovery has proven difficult. For example, barcode recoveries in the scale insects (Hemiptera, superfamily Coccoidea) are so low [7] that it has been suggested that an identification system for this group must be developed using another gene. Past efforts to overcome this problem have tried to design primer sets that bind within the COI gene. In the present study, we adopt an alternate approach, one in which the search for primer sites is focused on the tRNA genes that lie upstream of the COI gene in most arthropod mitochondrial genomes. Because these genes have several highly conserved sequence blocks, they have been used as an attractive target for primer design $[8,9]$. How- 
ever, there are two major complications that might impede such usage. First, there are a lot of sequence diversities in the conserved blocks of tRNA genes and only short sequences are actually conserved. So, previously developed primers often failed amplify target gene. Another one is the tRNA gene arrangement and its orientation. Individual mitochondrial genes occasionally move from one position in the mitochondrial genome to another. And also their orientation varies from forward to reverse. These difficulties lead to disruption of the design of primers with broad effectiveness. In this study, we strive to identify a tRNA gene whose position and orientation are relatively stable and which represent universality than previously developed primer. Applications of developed primer for COI barcoding the Hexapoda were also discussed.

\section{Results and Discussion}

\section{Mitochondrial genome analysis, primer design and} efficiency test

We began by analyzing gene arrangements for all of the arthropod mitochondrial genomes present in the Mitome database on April 10, 2009 [10]. This analysis re-confirmed the conserved gene arrangement of the three tRNA genes, tRNA-W, tRNA-C and tRNA-Y, ordinarily located upstream of COI in Hexapoda [9]. The position of tRNA-W was particularly consistent as it was found upstream of COI in 120 of 126 hexapod genomes and it was always oriented in a forward direction (Table 1). The six taxa lacking a tRNA-W gene in this position included 3 Phthiraptera, 1 Thysanoptera, 1 Pscoptera and 1 Lepidoptera. The same gene arrangement was also frequently apparent in the mitochondrial genomes of other arthropod subphyla. One or two additional tRNA genes $(\mathrm{C}, \mathrm{Y})$ were often present between tRNA-W and the start codon for COI. However, because each of these tRNA genes was only 60-68 bp in length, the tRNA-W gene was invariably located within $200 \mathrm{bp}$ upstream of COI, making it a good target for primer placement.

Alignment of the tRNA-W sequence from the 120 hexapods revealed two distinct groups of tRNA-W with high internal homogeneity in a central segment of the sequence (Figure 1). The first group (AAACTAWNARCCTTCAAAG) was dominant as it included 92 of the 120 lineages, while the remaining 28 lineages had a slightly divergent sequence (AAACTANWRATYTTCAAAATY). The former group have high sequence homology to previously developed tRNA-W primer, TW-J1301 [9] but the latter showed differences in their central six nucleotides, NWRATY-. This diversity may explain the previous failure of the tRNA-W primer. Two primers, tRWF1 and tRWF2, designed from these conserved sequences, were performance tested against DNA extracts from 141 species representing 121 different arthropod families in combination with a standard reverse primer (LepR1). As expected for hexapods, there was higher PCR success (Figure 2) for tRWF1 (74\%) than for tRWF2 (28\%). About $15 \%$ of DNA extracts were amplified by both primer sets, while amplification success rose to $87 \%$ when the two primers were combined in a 1:1 cocktail. Although this value was slightly lower than the success rate (90\%) with the usual arthropod primer set (LepF1, LepR1) the tRWF1\&2 cocktail amplified 11 of the 16 samples in our test set that failed with standard primers (Additional file 1 ). We also examined another universal primer for COI barcoding, LCO1490 and HCO2198 [11], and the PCR recoveries were very similar to that of LepF1/LepR1 primer (data not shown). Because of this complementarity, over $98 \%$ success in recovery of a barcode amplicon can be anticipated across all Hexapoda by two PCR reactions, the first using standard primers and the second (for those that failed in the first reaction) employing the primer set developed in this study. The 25 mer primer, TW-J1301, also showed partial complementarity how-

Table 1: Mitochondrial gene arrangements upstream of the COI gene

\begin{tabular}{|c|c|c|c|}
\hline Phylum & Subphylum & Mitochondrial gene arrangement ${ }^{a}$ & Arrangement identified ${ }^{b}$ \\
\hline \multirow[t]{4}{*}{ Arthropoda } & Chelicerata & $\mathbf{W},-\mathbf{C},-\mathbf{Y}, \mathrm{d}, \mathrm{e} ; \mathrm{W},-\mathrm{C},-\mathrm{Y},-Y ; W_{,}-\mathrm{C} ; \mathrm{W},-Y ; W$ & $33 / 41$ \\
\hline & Crustacea & $\mathbf{W},-\mathbf{C},-\mathbf{Y} ; \mathrm{W},-\mathrm{Q},-\mathrm{C}(-\mathrm{Y}) ; \mathrm{W}, \mathrm{Y} ; \mathrm{W}$ & $29 / 39$ \\
\hline & Hexapoda & $\mathbf{W},-\mathbf{C},-\mathbf{Y} ; \mathrm{W},-Y ; \mathrm{W},-\mathrm{C} ; \mathrm{W}$ & $120 / 126$ \\
\hline & Myriapoda & $\mathbf{W},-\mathbf{C} ; \mathbf{W}, \mathrm{W},-Y ; \mathrm{W}, \mathrm{C},-\mathrm{Y}$ & $8 / 8$ \\
\hline Chordata & Vertebrata & $\mathbf{W},-A,-N,-C,-Y$ & $60 / 60$ \\
\hline
\end{tabular}

a, All gene arrangement data are from Mitome http://www.mitome.info/

b, Proportion of taxa possessing one of the gene arrangements shown. For example, all 60 vertebrates share one gene order in which four other tRNA genes lie between tRNA-W and COI. By comparison 120 of 126 Hexapoda lineages possess one of four arrangements, none involving more than two intervening tRNA genes

c, Dominant gene arrangement is shown in bold

d, Reversed gene arrangements are represented as (-)

e, Abbreviations for tRNA genes: W - Tryptophan, C-Cysteine, Y-Tyrosine, Q - Glutamine, N-Asparagine and A- Alanine 


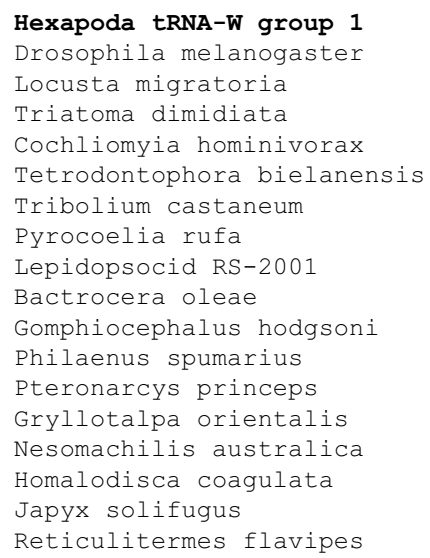

\section{Hexapoda tRNA-W group 2}

Apis mellifera ligustica Bombyx mori

Ostrinia nubilalis

Thrips imaginis

Melipona bicolor

Aleurodicus dugesii

onychiurus orientalis

Schizaphis graminum

Ruspolia dubia

Acyrthosiphon pisum

Bombus hypocrita

Trialeurodes vaporariorum

Phthonandria atrilineata

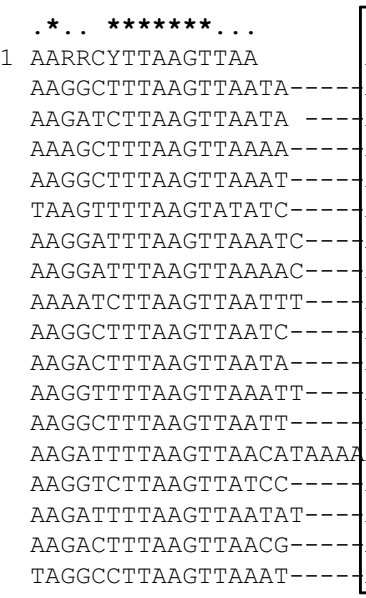

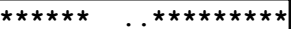

AAACTAWNARCCTTCAAAG

AAACTAATAACCTTCAAAG CTATAAATAAAGAAATT----TCTTTAAGCCTTA AAACTAATAACCTTCAAAGTTATAATTAAAAGTTATT---CTTTTAGGTCTTA AAACTATTAACCTTCAAAGTTAAAATTACAGGTATTAA----TGTAAGCTTTA AAACTAATAGCCTTCAAAGCTATAAATATAAGAATGAT--CTTTTAAGCCTTA AAACTAGGAGCCTTCAAAGCCCAAATAATAAGTAAAATC--TTGTAAACTTAA AAACTAACAACCTTCAAAGTGTAAATAGAATATTTCT----TCTAAGCCTTA AAACTAGTAACCTTCAAAGTTGCCAATAAAG-GATA------TTAAGCCTTA AAACTAATAACCTTCAAAGTATATATAAAT-TTATA---TATTTAGATTTTA AAACTAATAGCCTTCAAAG CTATAAATATAAGTTTAAT--CTTTTAAGCCTTA AGACTATTAGCCTTCAAAGCTTTAAATATAAGTTTAAT--CTTTTAAGTTTTA AAACTATTAACCTTCAAAGTTAAATATATAATATTT------TATAAGCCTTA AAACTAATAGCCTTCAAAG TTGTAAATAAAGTATCTA---TCTTTAAGCCTTA AAACTAATAGCCTTCAAAG TTGGAAATAAAGCAACT-------TTAAGTCTTA AAACTAATAGCCTTCAAAGCTATAAATAAAA-CTAAT---CTTTTAGACTTTA AAACTATTAACCTTCAAAGTTAAAAATGCTTTAAAG-------GTAAGTCTTA AAACTAATATCCTTCAAAGCTATCAATG-AAAGAAGAC-CTTTTAAGTCTTA AAACTAATAACCTTCAAAGCTATAAATA-AAGGGCCATAACCTTTAGGCCTTG

Figure 1 Alignment of tRNA-W genes from varied Hexapoda. All 126 Hexapod tRNA-W genes retrieved from GenBank were aligned and manually compared, revealing two groups with high internal sequence homogeneity. Sequence variation within representative species in each group is presented.

ever, it showed only 62\% PCR success. This primer has been designed from two conserved regions of tRNA-W gene $(7 \mathrm{mer}+18 \mathrm{mer})$ and the 18 mer-sequence is the same to tRWF1 primer without an additional $G$ on its 3 ' end. Generally, $G$ or $C$ base located at the 3 ' end of a PCR primer enhances PCR efficiency and this accounts for higher PCR success for tRWF1 primer than TW-J1301. Because the $t R N A$ primer set enables recovery of the COI sequence from the translation initiation codon, it provides insights into the cause for failed amplifications with the standard primer set, delivering the information needed to design a primer set that will amplify all hexapods.

\section{DNA barcoding analysis}

From the 125 successful PCR amplicons, 111 clean sequences were recovered. The presence of shorter, nonspecific amplicons was occasionally noted with the tRNA-W primer set, accounting for some failures in sequence recovery. The total length of the amplicons varied from $730 \mathrm{bp}-870 \mathrm{bp}$. The first $70-200 \mathrm{bp}$ of each read consisted of the sequences for one or more tRNA genes which were originated from different gene arrangement were excised during sequence editing. The remainder of most sequences (107) provided enough coverage for the COI gene to gain formal barcode status (512-711 bp), but four sequences were shorter $(279-497 \mathrm{bp})$. All sequences included $29 \mathrm{bp}-59 \mathrm{bp}$ of sequence information for the far 5 ' end of the COI gene that was not recovered with the standard primer set, reflecting the sequence region from the presumed initiation codon to the 3 ' end of the LepF1 primer binding region.

In mitochondria, six kinds of codon, ATN, TTG and GTG have been reported as a canonical translation initiation codon in vertebrate and insect. However, there are several exceptions to this rule, especially in COI gene. Previous reports demonstrated that quadruplets, such as ATAA, TTAA, TTAG, and ATTA could be used as a initiation signal [12-14] although these days, TCG or CGA has more convincing evidence than quadruplets, especially in Diptera [15] and Lepidoptera [16], respectively. In this study, 70 of the 111 sequences began with one of six known arthropod initiation codons (58 ATN, 14 TTG 


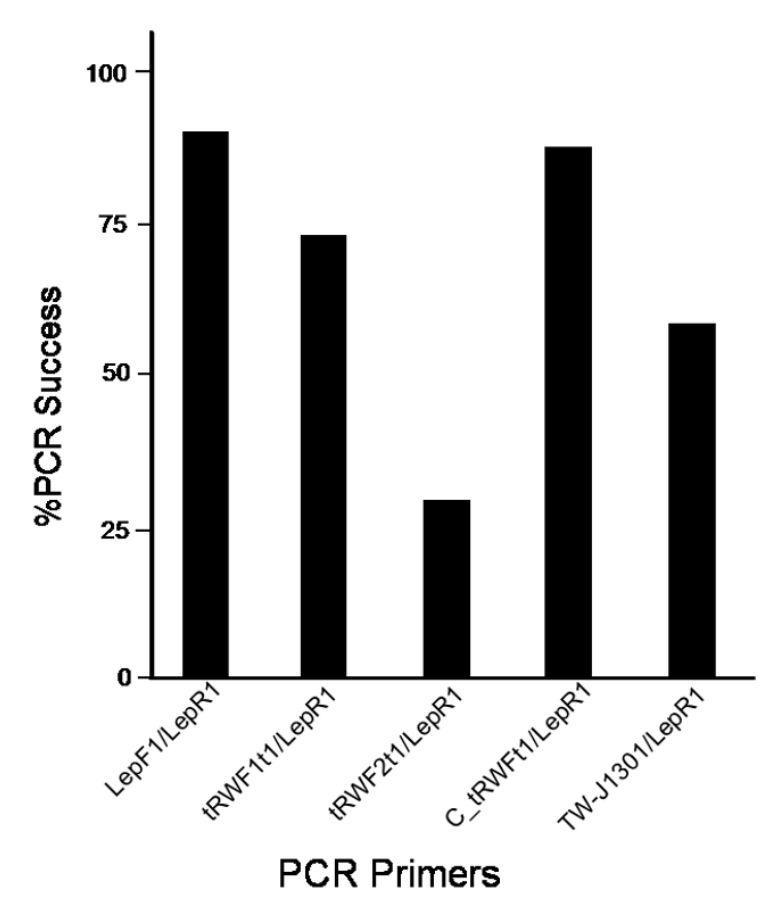

Figure 2 Amplification success for $\mathrm{CO} 1$ using standard primers (LepF1/LepR1) and two new forward primers positioned in the tRNA-W gene in combination with a standard reverse primer (LepR1) both separately and as a cocktail. TW-J1301 is previously reported tRNA-W primer [9].

and 1 GTG) and predicted 22 cases, mostly found in Diptera $(19 / 22)$ with two cases from a Orthoptera (1/1) and a Coleoptera (1/19), using TCG (including a CCG) codon (Additional file 2). Another possible initiator, CGA codon, were found in 17 species distributed in Lepidoptera (9/12), Ephemeroptera (2/5), Diptera (1/22), Mantodea (1/1), Trichoptera (1/5), and a Plecoptera (1/6). These are plausible because a TAG or a TAA stop codon presents at the beginning region of the COI gene and no canonical initiator was found within $30 \mathrm{bp}$ downstream from the stop codon. For two other sequences, the initiation codon was uncertain because sequencing results lacked bidirectional coverage at the 5 ' end because of presence of short non-specific amplicon which open found at the PCR reaction that used tailed primers.

No indels creating a frame shift or stop codons were present in the COI sequences, suggesting that none derived from a NUMTs. The fact that amplicons were longer than those generated by the standard primer set likely decreased the risk of NUMTs amplification because the latter are usually short $[17,18]$. Final sequencing success rate varied among the orders (Figure 3). Lepidoptera, Ephemeroptera and Plecoptera showed 100\% success ( $n=$ 12,6 , and 6, respectively), while Hymenoptera showed only $41 \%$ sequencing success from 17 samples because PCR generated only 7 clear amplicons. The rest showed

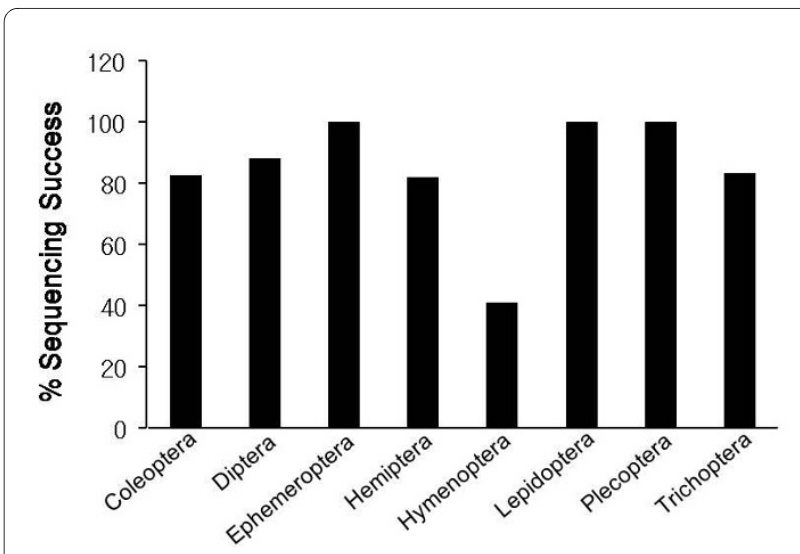

Figure 3 Success in recovering high quality sequence records from members of $\mathbf{8}$ insect orders represented by $\mathbf{6}$ or more different families. The number of families examined varied among orders: Coleoptera (23), Diptera (25), Ephemeroptera (6), Hemiptera (11), Hymenoptera (17), Lepidoptera (12), Plecoptera (6) and Trichoptera (6).

weak, non-specific or no amplification (Additional file 1lane F3 to G7). Diptera, Trichoptera, Hemiptera and Coleoptera ( $n=25,6,11$ and 23, respectively) showed 82$88 \%$ success. The value for the Hemiptera excluded results for the family Pseucococcidae which only showed $50 \%$ success $(n=6)$. The very low PCR and sequencing success in the Hymenoptera, which showed $88 \%$ PCR success with the standard primer set, implies high diversity in the location, orientation or sequence of the tRNAW gene in members of this order, a result already reported for one family of hymenopterans- the Pompilidae [19].

Sequence analysis of 11 taxa which failed to amplify with standard primers indicated that such failures occurred when more than three nucleotides were substituted and usually involved one or more substitutions within 10 nucleotides of the 3 ' end (Figure 4a). Most of these substitutions were synonymous, but changes causing amino acid substitutions were detected in the three scale insects, $P$. aceris, $P$. kraunhiae and P. comstocki. The GTA transition at the first position of two codons provoked the substitution of GlyTSer and of AspTAsn at sites near the 3 ' end of the primer binding region. These otherwise undetected substitutions explain past failures to recover amplicons for the barcode region in these scale insects using standard primers. We tried to confirm that these substitutions are popular for scale insect species through designing a new forward primer, PcoF1, which reflecting the substitutions and testing its performance for 28 scale insect species. The newly designed primer showed successive amplification for 26 of 28 scale insects samples when combined with the LepR1 primer (Figure 5) while the universal primer sets could only recover 5-6 clear amplicons and the cocktail primer generated ampli- 


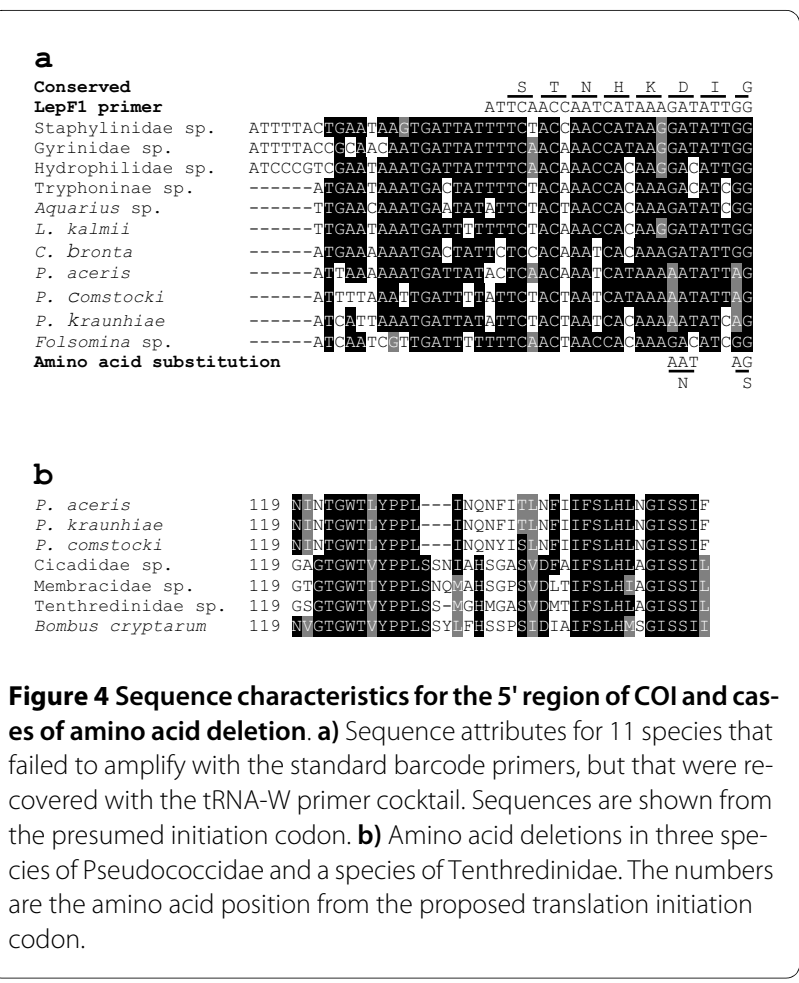

cons only in Pseucococcidae samples. The 26 successful amplicons includes: 9 Pseudococcidae, 9 Diaspididae, 6 Coccidae, an Ortheziidae and a Conchaspididae species (Additional file 3). The present results confirm the feasibility of creating a comprehensive barcode library for scale insects which previously known as 'difficult barcode group'.

Two additional samples, deriving from species of the wasp family Ichneumonidae (Tryphoninae sp.) and the collembolan family Isotomidae (Folsomina $\mathrm{sp}$.), each possessing three TTC substitutions near the 3 ' end of the primer binding region, represent the only barcode records so far reported for these lineages. The nearest homology group to the species of Tryphoniinae had just $87.6 \%$ similarity, while the nearest sequence to the species of Folsomina showed just $82.4 \%$ congruence.

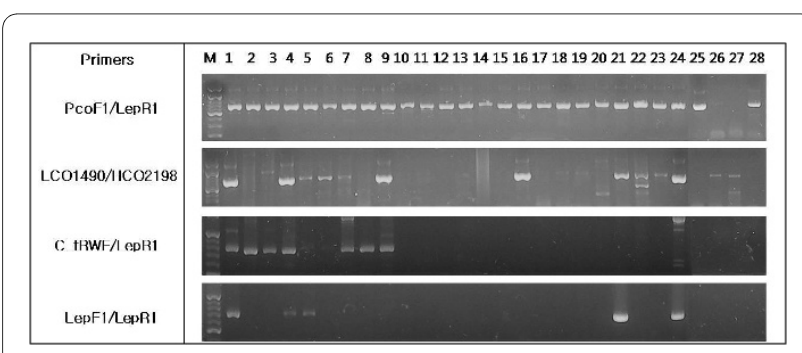

Figure $\mathbf{5}$ Gel images of PCR amplicons for $\mathbf{2 8}$ scale insect species. The samples included 9 Pseudococcidae (1-9), 9 Diaspididae (10-18), 6 Coccidae (19-24), 1 Ortheziidae, 1 Eriococcidae, 1 Margarodidae and 1 Conchaspididae.
The alignment of all 111 sequences revealed five cases of amino acid deletion. A block of three amino acids were deleted in three species of Pseudococcidae (Figure 4b). This same deletion occurs in all members of scale insect families tested in this study representing the first case of a three amino acid deletion in COI across all Hexapoda that have been analyzed. Interestingly, there was a single amino acid deletion in a species belonging to the hymenopteran family, Tenthredinidae, at a similar position. A two amino acid deletion was found in a species of Pompilidae (Hymenoptera), but it occurred at a different position in the standard COI barcode region (amino acid position 175 from the initiation codon versus 131/133 amino acids for the scale insects and the tenthrendinid).

As already noted, the new primer cocktail failed to amplify the barcode region in some Hexapoda. Its failure in these cases was likely due to shifts in position of the tRNA-W gene, the existence of intergenic gap between tRNA-W and COI or to sequence variation in the segment of tRNA-W targeted for primer binding. However, this cocktail primer can be a useful supplementary tool for standard COI barcoding method. For example, we could attain $94 \%$ of successful barcode from over a thousand of Hemiptera specimens which represents over 200 species with only two PCR amplification steps; the first run, performed with standard primer, obtained only $82 \%$ successes. Then the second run conducted with cocktail primer against samples failed at first run (unpublished data). Additionally, this cocktail primer can reduce barcoding failures caused by unexpected amplification of endosymbiont COI where occasionally found in barcoding the Hemiptera.

\section{Conclusions}

The tRNA-W primer cocktail developed in this study successfully amplified the DNA barcode region for most hexapods including many species which failed to generate an amplicon with the standard primer set. Use of this cocktail not only improves the success of barcode recovery, but also provides the information needed to design a primer set for problem groups. The current standard primer set for hexapods are not degenerate, so further study of the upstream sequence of COI promises to aid the development of a new primer cocktail with higher generality.

\section{Methods}

Mitochondrial genome analysis and primer design

All mitochondrial genome arrangements were assessed using the gene arrangement comparison tool on Mitome, the Mitochondrial Genome Database http://www. mitome.info. tRNA-W gene sequences were retrieved from GenBank and aligned by Clustal W [20]. Some manual modification was done to divide two distinct groups 
of tRNA-W with high internal homogeneity. Two forward PCR primers: tRWF1 (5'-AAACTAATARCCTTCAAAG3') and tRWF2 (5'-AAACTAATAATYTTCAAAATTA3') with M13 tails (5'-TGTAAAACGACGGCCAGT-3') on their $5^{\prime}$ end were designed to best represent these two groups and were combined with a standard reverse primer (LepR1) to produce a cocktail (1:1 ratio) which was tested against 141 species representing 121 different families of Hexapoda. A new forward primer for scale insects, PcoF1: 5'- CCTTCAACTAATCATAAAAAT ATYAG - 3', was designed reflecting sequence diversity in scale insects at the same positions for universal COI barcoding primers.

\section{DNA analysis}

Most of the specimens used in this study derived from collections made by BIO researchers at sites in North America over the past two years. Six scale insect samples were provided from the Central Post-entry Quarantine Station in South Korea. Jointly, these collections included 141 species representing 121 different families of Hexapoda (Additional file 4). All DNA was extracted from either dried or ethanol-fixed leg samples using a standard Glass Fibre extraction protocol [21] except for some small specimens which were processed as whole individuals. PCR thermocycling was done under the following conditions: $2 \mathrm{~min}$ at $95^{\circ} \mathrm{C} ; 5$ cycles of $40 \mathrm{sec}$ at $94^{\circ} \mathrm{C}, 40 \mathrm{sec}$ at $45^{\circ} \mathrm{C}, 70 \mathrm{sec}$ at $72^{\circ} \mathrm{C} ; 40$ cycles of $40 \mathrm{sec}$ at $94^{\circ} \mathrm{C}, 40 \mathrm{sec}$ at $51^{\circ} \mathrm{C}, 70 \mathrm{sec}$ at $72^{\circ} \mathrm{C} ; 5 \mathrm{~min}$ at $72^{\circ} \mathrm{C}$; held at $4^{\circ} \mathrm{C}$.

PCR, PCR check and DNA sequencing were carried out using standard methods[22]. Contigs were assembled using CodonCode aligner Ver2.0.6 (CodonCode Co.) and were subsequently aligned by the same software. The locations of the deletions were identified by manual editing. All sequences have been deposited in GenBank and accession numbers (GU013562 $\sim$ GU013672 and GU936932 GU936957), for the sequences, as well as specimen and collection data, and trace files are available within the DIMC project files in BOLD http:// www.barcodinglife.org.

\section{Additional material}

Additional file 1 PCR comparisons. Agarose gels comparing the PCR products generated using both the standard primer set and the tRNA-W primer cocktail.

Additional file $\mathbf{2}$ Initiation codon of the COI. Identification of possible initiation codons for sequences amplified by the tRNA-W cocktail was analyzed.

Additional file 3 Scale insect samples used in this study

Additional file 4 Information on samples used in this study.

\section{Authors' contributions}

DSP performed laboratory work, led project design and data analysis and cowrote the manuscript. PDNH aided in design of the project, provided tools/ reagents, and co-wrote the manuscript. HWO aided in design of the project and helped in laboratory work. SJS collected and identified the scale insect samples and helped in laboratory work. All authors read and approved the final manuscript.

\section{Acknowledgements}

We thank Jayme Sones, David Porco and Xin Zhou for providing specimens or DNA extracts. We are also grateful to Justin Schonfeld and Robert Ward for comments on an earlier draft of this manuscript. This research was supported by a grant (FDM0401012) from the National Plant Quarantine Service to D-S Park and by grants from Genome Canada through the Ontario Genomics Institute to PDNH.

\section{Author Details}

1Biological Resource Center, Korea Research Institute of Bioscience \& Biotechnology, Daejeon 305-806, Korea, 2Industrial Biomaterials Research Center, Korea Research Institute of Bioscience \& Biotechnology, Daejeon 305806, Korea, ${ }^{3}$ Youngnam Regional Office, National Plant Quarantine Service, Busan 600-016, Korea and 4Biodiversity Institute of Ontario, University of Guelph, Guelph, Ontario, N1G 2W1, Canada

Received: 26 March 2010 Accepted: 9 July 2010

Published: 9 July 2010

\section{References}

1. Hebert PDN, Cywinska A, Ball SL, deWaard JR: Biological identifications through DNA barcodes. Proc Biol Sci 2003, 270(1512):313-321.

2. Hebert PDN, Gregory TR: The promise of DNA barcoding for taxonomy. Syst Biol 2005, 54(5):852-859.

3. Hajibabaei M, Janzen DH, Burns JM, Hallwachs W, Hebert PDN: DNA barcodes distinguish species of tropical Lepidoptera. Proc Natl Acad Sci USA 2006, 103(4):968-971.

4. Ward RD, Zemlak TS, Innes BH, Last PR, Hebert PDN: DNA barcoding Australia's fish species. Philos Trans R Soc Lond B Biol Sci 2005, 360(1462):1847-1857.

5. Sevilla RG, Diez A, Noren M, Mouchel O, Jerome M, Verrez-Bagnis V, van Pelt H, Favre-Krey L, Bautista JM: Primers and polymerase chain reaction conditions for DNA barcoding teleost fish based on the mitochondrial cytochrome $b$ and nuclear rhodopsin genes. Mol Ecol Notes 2007, 7:730-734.

6. Smith MA, Wood DM, Janzen DH, Hallwachs W, Hebert PDN: DNA barcodes affirm that 16 species of apparently generalist tropical parasitoid flies (Diptera, Tachinidae) are not all generalists. Proc Natl Acad Sci USA 2007, 104(12):4967-4972.

7. Kondo T, Gullan PJ, Williams DJ: Coccidology. The study of scale insects (Hemiptera: Sternorrhyncha: Coccoidea). Revista Corpoica- Cienciay Technologia Agropecuaria 2008, 9(2):55-61.

8. Lewis RL, Beckenbach AT, Mooers AO: The phylogeny of the subgroups within the melanogaster species group: likelihood tests on $\mathrm{COI}$ and COIl sequences and a Bayesian estimate of phylogeny. Mol Phylogenet Evol 2005, 37(1):15-24.

9. Simon C, Buckley TR, Frati F, Stewart JB, Beckenbach AT: Incorporating molecular evolution into phylogenetic analysis, and a new compilation of conserved polymerase chain reaction primers for animal mitochondrial DNA. Annu Rev Ecol Evol 2006, 37:545-579.

10. Lee YS, Oh J, Kim YU, Kim N, Yang S, Hwang UW: Mitome: dynamic and interactive database for comparative mitochondrial genomics in metazoan animals. Nucleic acids research 2008:D938-942.

11. Folmer O, Black M, Hoeh W, Lutz R, Vrijenhoek R: DNA primers for amplification of mitochondrial cytochrome coxidase subunit I from diverse metazoan invertebrates. Mol Mar Biol Biotechnol 1994, 3(5):294-299.

12. Clary DO, Wolstenholme DR: Genes for cytochrome c oxidase subunit I, URF2, and three tRNAs in Drosophila mitochondrial DNA. Nucleic acids research 1983, 11(19):6859-6872.

13. de Bruijn MH: Drosophila melanogaster mitochondrial DNA, a novel organization and genetic code. Nature 1983, 304(5923):234-241.

14. Lunt DH, Zhang DX, Szymura JM, Hewitt GM: The insect cytochrome oxidase I gene: evolutionary patterns and conserved primers for phylogenetic studies. Insect Mol Biol 1996, 5(3):153-165.

15. Krzywinski J, Grushko OG, Besansky NJ: Analysis of the complete mitochondrial DNA from Anopheles funestus: an improved dipteran 
mitochondrial genome annotation and a temporal dimension of mosquito evolution. Mol Phylogenet Evol 2006, 39(2):417-423.

16. Kim MI, Baek JY, Kim MJ, Jeong HC, Kim KG, Bae CH, Han YS, Jin BR, Kim I: Complete nucleotide sequence and organization of the mitogenome of the red-spotted apollo butterfly, Parnassius bremeri (Lepidoptera: Papilionidae) and comparison with other lepidopteran insects. Mol Cells 2009, 28(4):347-363.

17. Pereira SL, Baker AJ: Low number of mitochondrial pseudogenes in the chicken (Gallus gallus) nuclear genome: implications for molecular inference of population history and phylogenetics. BMC Evol Biol 2004, 4:17.

18. Song H, Buhay JE, Whiting MF, Crandall KA: Many species in one: DNA barcoding overestimates the number of species when nuclear mitochondrial pseudogenes are coamplified. Proc Natl Acad Sci USA 2008, 105(36):13486-13491.

19. Szafranski P: The mitochondrial trn-cox1 locus: rapid evolution in Pompilidae and evidence of bias in cox 1 initiation and termination codon usage. Mitochondrial DNA 2009, 20(1):15-25.

20. Thompson JD, Higgins DG, Gibson TJ: CLUSTAL W: improving the sensitivity of progressive multiple sequence alignment through sequence weighting, position-specific gap penalties and weight matrix choice. Nucleic acids research 1994, 22(22):4673-4680.

21. Ivanova NV, deWaard JR, Hebert PDN: An inexpensive, automationfriendly protocol for recovering high-quality DNA. Molecular Ecology Notes 2006. doi:10.1111/j.1471-8286.2006.01428.x

22. Fisher BL, Smith MA: A revision of Malagasy species of Anochetus mayr and Odontomachus latreille (Hymenoptera: Formicidae). PLoS One 2008, 3(5):e1787.

doi: 10.1186/1471-2164-11-423

Cite this article as: Park et al., Recovery of the mitochondrial COI barcode region in diverse Hexapoda through tRNA-based primers BMC Genomics 2010, 11:423

Submit your next manuscript to BioMed Central and take full advantage of:

- Convenient online submission

- Thorough peer review

- No space constraints or color figure charges

- Immediate publication on acceptance

- Inclusion in PubMed, CAS, Scopus and Google Scholar

- Research which is freely available for redistribution

Submit your manuscript at www.biomedcentral.com/submit
Ciomed Central 